\title{
ASTROPHYSICS
}

DOI: http://dx.doi.org/10.18524/1810-4215.2017.30.117154

\section{ZIRCONIUM ABUNDANCES IN THE CENTRAL PART OF THE dSph FORNAX GALAXY}

\author{
S. M. Andrievsky ${ }^{1,2}$, S. A. Korotin ${ }^{1,4}$, V. Hill $^{3}$, A. V. Zhukova ${ }^{4}$ \\ 1 Astronomical Observatory, Odessa National University, \\ Shevchenko Park, 65014 Odessa, Ukraine, andrievskii@ukr.net \\ ${ }^{2}$ GEPI, Observatoire de Paris, PSL, Research University, CNRS, Univ Paris Diderot, \\ Sorbonne Paris Cité, Place Jules Janssen, 92195 Meudon, France \\ ${ }^{3}$ Université Côte d'Azur, Observatoire de la Côte d'Azur, CNRS, Laboratoire Lagrange, \\ Bd de l'Observatoire, CS 34229, 06304, Nice Cedex 4, France \\ ${ }^{4}$ Crimean Astrophysical Observatory, Nauchny, 298409, Crimea
}

\begin{abstract}
We derived LTE zirconium abundance in a sample of 81 giant stars in dwarf spheroidal Fornax galaxy. The LTE synthesis was used for this aim. We obtained the lower Zr abundance in Fornax galaxy comparing to the relevant value in the thick/thin disc of the Milky Way. We note that this result may be affected by the NLTE effects that decrease the real zirconium abundance.
\end{abstract}

Keywords: stars: abundances - galaxies: individual: Fornax - galaxies: evolution - galaxies: dwarf galaxies: formation

\section{Introduction.}

Dwarf spheroidal galaxy Fornax is one of the most luminous satellites of the Milky Way. Its stellar population was studied spectroscopically by many authors. In particular, Letarte et al. (2010) and Lemasle et al. (2014) determined abundances of $\alpha$ and some $s$-process elements in the giants of this stellar system. Among $s$-process elements zirconium was not studied in the stars of this galaxy. Therefore we decided to fill in this gap.

\section{Sample of the stars}

The sample of our program stars has been previously investigated by Letarte et al. (2010). The spectra were secured with ESO VLT facilities with resolving power of about 20000 in the two ranges: 5340-5620 $\AA$, and $6120-6701 \AA$. 81 RGB stars were selected in the central part of Fornax dSph galaxy. In this article we report the results of determination of zirconium abundnace. We used stellar parameters and iron abundance determined by Letarte et al. (2010). The

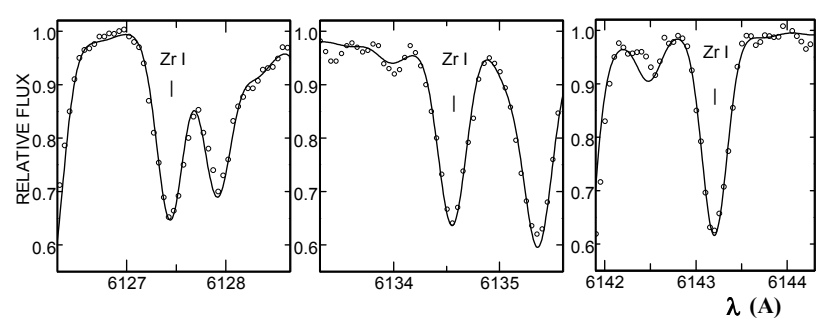

Figure 1: Synthetic and observed spectra of three zirconium lines for the star BL211 (the best zirconium abundance is $(\mathrm{Zr} / \mathrm{H})=2.14)$. Circles - observed spectrum, continuos line - synthetic spectrum.

program stars and their iron abundance are listed in Table 2 together with our LTE abundance of Zr (see next sections).

\section{Abundance determination details.}

As we have already mentioned Letarte et al. did not derive abundance of zirconium. From the light $s$ process element they give abundance only for yttrium. We performed LTE profile synthesis for the three Zr I lines: 6127, 6134 and $6143 \AA$ using SYNTHV code (Tsymbal 1996). Blending lines of other species were taking into account by using the Vienna Atomic Line Database (VALD, Kupka et al. 1999). ${ }^{1} \quad$ An example of the profile fitting is given in Fig. 1. Table 1 contains an information about zirconium line parameters used in our calculations. Zirconium abundance in our program stars is listed in Table 2 .

\footnotetext{
${ }^{1}$ http://vald.astro.univie.ac.at/ vald3/php/vald.php
} 
Table 1: Zirconium line parameters.

\begin{tabular}{ccc}
\hline El & $\lambda(\AA)$ & $\log$ gf \\
\hline \hline Zr I & 6127.48 & -1.06 \\
Zr I & 6134.59 & -1.28 \\
Zr I & 6143.25 & -1.10 \\
\hline
\end{tabular}

\section{Results and discussion.}

It is generally accepted that zirconium nuclei are produced in the higher-mass AGB stars (Prantzos et al. 1990, Raiteri et al. 1992). Production rate of the firstpeak (Sr, Y, Zr) elements depends also on the metallicity of the AGB star. Metal-poor AGB stars produce a larger amount of the $s$-process peak nuclei.

The ratio $[\mathrm{Zr} / \mathrm{Fe}]$ in our program stars appears to be below the value which is predicted by the Galaxy model of chemical evolution for the thick/thin disc (Bisterzo et al. 2017) (see Fig. 2).

Battistini \& Bensby (2016) having analysed 311 stars in the solar neighbourhood found an increase of zirconium abundance in the Galactic thin and thick disc with metallicity decrease. Their mean $[\mathrm{Zr} / \mathrm{Fe}]$ value in the metallicity region from about -1 to -0.5 is approximately $0.3-0.4$ dex which is significanly higher than the mean value from the Fornax star sample. On contrary, Reddy et al. (2003) analysed $181 \mathrm{~F}$ and $\mathrm{G}$ dwarfs from Galactic disc, and their result on zirconium shows that at least up to $[\mathrm{Fe} / \mathrm{H}] \approx-0.7$ there is no sign of an increase of abundance of this element with iron abundance decrease. The similar result was published also by Brewer \& Carney (2006). The situation with zirconium becomes even more entangled if we take into account that in the different studies the different $\mathrm{Zr}$ lines were used to derive abundance of this element (for instance, Battistini \& Bensby (2016) employed Zr I $4687 \AA$, $4739 \AA$, and Zr II $4208 \AA, 5112 \AA$ lines, while we used set of the red zirconium lines). Could such a discrepancy in the literature data be the result of ignoring of the NLTE corrections? There is a very limited information in the literature concerning the NLTE corrections for zirconium LTE abundance derived from different lines. Here one can mention only the paper of Velichko et al.(2010), who provided some limited data on corrections that should be applied to abundances derived from Zr I $4687 \AA$, Zr II $4208 \AA$, and $5112 \AA$ lines in cool giants. According to those authors corrections are of about $0.2-0.3 \mathrm{dex}$, and they should even increase the LTE abundances (later authors published erratum where they informed that they used wrong ionization potential for Zr I). Nevertheless, those authors say (but without quantitative estimates) that observed red zirconium lines should be weakened due to the NLTE effects, what means that our result on LTE zirconium

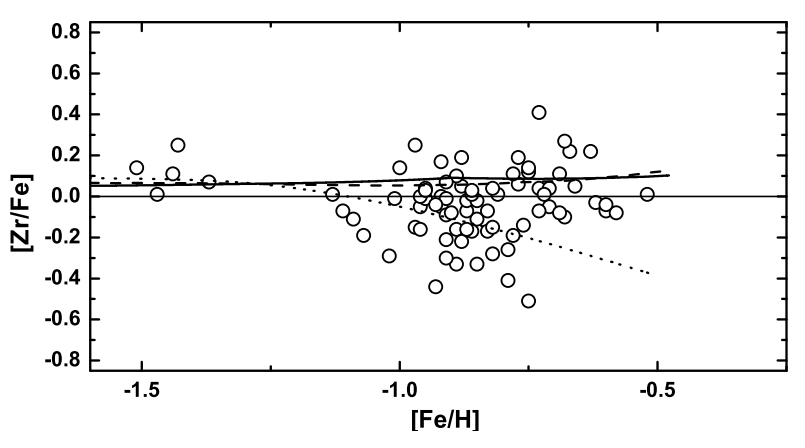

Figure 2: $[\mathrm{Zr} / \mathrm{Fe}]$ vs. $[\mathrm{Fe} / \mathrm{H}]$. Circles - our stars, the model data are from Bisterzo et al. (2017). Continuous line - thin disk, dashed line - thick disk, dotted line halo.

"underabundance" in the Fornax giants (Fig. 2) may be just an artefact. In our analysis we used Zr I 6127 , 6134 and $6143 \AA$, for which no information about NLTE effect exists in the literature. Taking into account that different zirconium lines were used by the different authors to derive abundance in dSph Fornax galaxy and in the Milky Way, and no reliable data on the NLTE corrections for zirconium abundance derived from those lines exist in the literature, we leave the question about the detected difference in zirconium abundance in Fornax galaxy and the Milky Way stars open.

Acknowledgements. We are thankful to Dr. Sara Bisterzo for her kind help with theoretical modeling data. SMA is thankful to the Universite Côte d'Azur, Observatoire de la Côte d'Azur, CNRS, Laboratoire Lagrange administration for their hospitality during his visit in 2016, and financial support, and also for the partial financial support from the SCOPES grant No. IZ73Z0-152485, which is also acknowledged by SAK.

\section{References}

Battistini C., Bensby T.: 2016, A\&A, 586, A49.

Bisterzo S., Travaglio C., Wiescher M., Käppeler F., Gallino R.: 2017, ApJ, 835, 97.

Brewer M.-M., Carney B.W.: 2006, AJ, 131, 431.

Busso M., Gallino R., \& Wasserburg G. J.: 1999, ARA $\& A$, 37, 239.

Kupka F., Piskunov N., Ryabchikova T. et al.: 1999, $A \xi A S, \mathbf{1 3 8}, 119$.

Lemasle B., de Boer T.J.L., Hill V., et al.: 2014, $A \& A, \mathbf{5 7 2}, \mathrm{A} 88$.

Letarte B., Hill V., Tolstoy E. et al.: 2010, AछA, 523, A17.

Prantzos N., Hashimoto M., Nomoto K.: 1990, A\&A, 234, 211.

Raiteri C.M., Gallino R., Busso M.: 1992, ApJ, 387, 263 . 
Table 2: Program stars, their metallicity and abundances of $\mathrm{Zr}$ and $\mathrm{Ba} . \quad(\mathrm{El} / \mathrm{H})=\log \epsilon(\mathrm{El})+12.00 ;[\mathrm{El} / \mathrm{H}]=$ $(\mathrm{El} / \mathrm{H})_{\text {star }}-(\mathrm{El} / \mathrm{H})_{\text {Sun }}$.

\begin{tabular}{|c|c|c|c|c|c|c|c|}
\hline Star & {$[\mathrm{Fe} / \mathrm{H}]$} & $(\mathrm{Zr} / \mathrm{H})$ & {$[\mathrm{Zr} / \mathrm{Fe}]$} & Star & {$[\mathrm{Fe} / \mathrm{H}]$} & $(\mathrm{Zr} / \mathrm{H})$ & {$[\mathrm{Zr} / \mathrm{Fe}]$} \\
\hline BL038 & -0.88 & 1.76 & 0.05 & BL185 & -0.71 & 1.92 & 0.04 \\
\hline BL045 & -1.09 & 1.39 & -0.11 & BL190 & -0.79 & 1.39 & -0.41 \\
\hline BL052 & -1.02 & 1.28 & -0.29 & BL195 & -0.97 & 1.87 & 0.25 \\
\hline BL065 & -1.43 & 1.41 & 0.25 & BL196 & -1.07 & 1.33 & -0.19 \\
\hline BL076 & -0.85 & 1.63 & -0.11 & BL197 & -0.89 & 1.54 & -0.16 \\
\hline BL077 & -0.79 & 1.54 & -0.26 & BL203 & -0.83 & 1.69 & -0.07 \\
\hline BL079 & -0.52 & 2.08 & 0.01 & BL204 & -1.00 & 1.73 & 0.14 \\
\hline BL081 & -0.62 & 1.94 & -0.03 & BL205 & -0.69 & 2.01 & 0.11 \\
\hline BL084 & -0.85 & 1.41 & -0.33 & BL208 & -0.66 & 1.98 & 0.05 \\
\hline BL085 & -2.59 & - & - & BL210 & -0.76 & 1.69 & -0.14 \\
\hline BL091 & -0.96 & 1.58 & -0.05 & BL211 & -0.67 & 2.14 & 0.22 \\
\hline BL092 & -0.95 & 1.63 & -0.01 & BL213 & -0.93 & 1.62 & -0.04 \\
\hline BL096 & -0.75 & 1.33 & -0.51 & BL216 & -0.77 & 1.88 & 0.06 \\
\hline BL097 & -0.92 & 1.67 & 0.00 & BL218 & -0.60 & 1.92 & -0.07 \\
\hline BL100 & -0.93 & 1.22 & -0.44 & BL221 & -0.86 & 1.74 & 0.01 \\
\hline BL104 & -0.96 & 1.63 & 0.00 & BL227 & -0.91 & 1.75 & 0.07 \\
\hline BL113 & -0.75 & 1.96 & 0.12 & BL228 & -0.88 & 1.49 & -0.22 \\
\hline BL115 & -1.47 & 1.13 & 0.01 & BL229 & -0.71 & 1.83 & -0.05 \\
\hline BL123 & -0.97 & 1.47 & -0.15 & BL233 & -0.68 & 1.81 & -0.10 \\
\hline BL125 & -0.73 & 1.90 & 0.04 & BL239 & -0.91 & 1.47 & -0.21 \\
\hline BL132 & -0.89 & 1.37 & -0.33 & BL242 & -1.11 & 1.41 & -0.07 \\
\hline BL135 & -0.95 & 1.68 & 0.04 & BL247 & -0.82 & 1.62 & -0.15 \\
\hline BL138 & -1.01 & 1.57 & -0.01 & BL250 & -0.68 & 2.18 & 0.27 \\
\hline BL140 & -0.87 & 1.65 & -0.07 & BL253 & -0.73 & 1.79 & -0.07 \\
\hline BL141 & -0.82 & 1.49 & -0.28 & BL257 & -0.58 & 1.93 & -0.08 \\
\hline BL146 & -0.92 & 1.62 & -0.05 & BL258 & -0.60 & 1.95 & -0.04 \\
\hline BL147 & -1.37 & 1.29 & 0.07 & BL260 & -0.87 & 1.56 & -0.16 \\
\hline BL148 & -0.63 & 2.18 & 0.22 & BL261 & -0.86 & 1.76 & 0.03 \\
\hline BL149 & -0.91 & 1.59 & -0.09 & BL262 & -0.78 & 1.92 & 0.11 \\
\hline BL150 & -0.83 & 1.59 & -0.17 & BL266 & -1.44 & 1.26 & 0.11 \\
\hline BL151 & -0.86 & 1.56 & -0.17 & BL267 & -0.72 & 1.88 & 0.01 \\
\hline BL155 & -0.75 & 1.98 & 0.14 & BL269 & -0.81 & 1.79 & 0.01 \\
\hline BL156 & -1.13 & 1.47 & 0.01 & BL278 & -0.73 & 2.27 & 0.41 \\
\hline BL158 & -0.87 & 1.70 & -0.02 & BL279 & -1.51 & 1.22 & 0.14 \\
\hline BL160 & -0.95 & 1.67 & 0.03 & BL295 & -0.69 & 1.82 & -0.08 \\
\hline BL163 & -0.77 & 2.01 & 0.19 & BL300 & -0.92 & 1.84 & 0.17 \\
\hline BL166 & -0.89 & 1.80 & 0.10 & BL304 & -0.96 & 1.47 & -0.16 \\
\hline BL168 & -0.88 & 1.90 & 0.19 & BL311 & -0.78 & 1.62 & -0.19 \\
\hline BL171 & -0.90 & 1.61 & -0.08 & BL315 & -0.82 & 1.81 & 0.04 \\
\hline BL173 & -0.85 & 1.72 & -0.02 & BL323 & -0.91 & 1.38 & -0.30 \\
\hline BL180 & -0.91 & 1.67 & -0.01 & & & & \\
\hline
\end{tabular}

Reddy B.E., Tomkin J., Lambert D.L., Allende Prieto

C.: 2003, MNRAS, 340, 304.

Tsymbal V.V.: 1996, Model Atmosph. and Spectr.

Synth., eds.: S. J. Adelman, F. Kupka, W.W. Weiss,

San Francisco, ASP Conf. Ser., 108.

Velichko A., Mashonkina L., Nilsson H.: 2010, AstL,

36, 664 . 